\title{
Thermoelectric behavior of hot collisional and magnetized QCD medium from an effective kinetic theory
}

\author{
Manu Kurian* \\ Indian Institute of Technology Gandhinagar, Gandhinagar-382355, Gujarat, India
}

(Received 4 February 2021; accepted 26 February 2021; published 26 March 2021)

\begin{abstract}
In this paper, we study the thermoelectric behavior of the quark-gluon plasma within the framework of an effective kinetic theory. We adopt a quasiparticle model to incorporate the effects of a thermal medium. The thermoelectric response of the medium is quantified in terms of the Seebeck and Nernst coefficient. Utilizing the relaxation time approximation and Bhatnagar-Gross-Krook collision kernels in an effective Boltzmann equation, we compute the dependence of the Seebeck coefficients on collision temperature and quark chemical potential. We furthermore explore the magnetic field dependence of the thermoelectric effects in a weakly and strongly magnetized medium. The thermoelectric response of a hot QCD medium is shown to be most prominent at low temperatures close to the transition temperature.
\end{abstract}

DOI: $10.1103 /$ PhysRevD.103.054024

\section{INTRODUCTION}

Experimental programs at Relativistic Heavy Ion Collider (RHIC) and Large Hadron Collider (LHC) have confirmed the existence of a hot and dense state of nuclear matter known as the quark-gluon plasma (QGP) $[1,2]$. The space-time evolution of the QGP has been successfully described within the framework of relativistic dissipative hydrodynamics $[3,4]$. The nonequilibrium physics of the created medium critically depends on its transport coefficients associated with the momentum, thermal and electric charge transport processes. Besides quantifying the dissipative processes and system responses to electromagnetic fields, these transport coefficients act as input parameters for the hydrodynamical approach. The relevance of transport parameters for the quantitative description of the measured observable has been explored in the collision experiments at the RHIC and LHC [5-8].

Recently, the LHC and RHIC have reported that the directed flow $v_{1}$ of $D$ and $\bar{D}^{0}$ mesons are about 3 orders of magnitude higher than that of the charged hadrons $[9,10]$. These observations indicate the existence of a strong magnetic field in the initial stages of the heavy-ion collision. The theoretical estimations of the strength of the magnetic field in the primary stage of collision are in the order of $(1-15) m_{\pi}^{2}[11,12]$ and have generated wide enthusiasm. The impact of the magnetic field on anomalous transport

\footnotetext{
*manu.kurian@iitgn.ac.in
}

Published by the American Physical Society under the terms of the Creative Commons Attribution 4.0 International license. Further distribution of this work must maintain attribution to the author(s) and the published article's title, journal citation, and DOI. Funded by SCOAP. phenomena [13,14], heavy quark dynamics $[15,16]$, quarkonia suppression [17,18], electromagnetic probes [19], jet quenching [20], transport and thermodynamic of QCD medium [21-23] has gained much attention recently. Studies have shown that the lifetime of the magnetic field in the QGP may depend on the medium properties [24,25], which indicates that the magnetic field may persist in the medium for a longer time than expected. Owing to the fact that the decay of the magnetic field is not completely modeled yet, the hot QCD medium properties have been explored both in the strong and weak magnetic field regimes. In a strongly magnetized medium $\sqrt{q_{f} B} \gg T$, where $q_{f} B$ is the strength of the field and $T$ is the temperature of the medium, the charged fermion motion is constrained along the direction of the magnetic field via Landau quantization. On the other hand, in the presence of a weak magnetic field $\sqrt{q_{f} B} \ll T$, cyclotron frequency captures the magnetic field effects.

A temperature gradient over the extent of the fluid results in thermal dissipation in the hot QCD medium. The thermal transport in the QGP medium has been explored within kinetic theory framework [26,27], effective models [28-31] and Kubo formalism [32]. Similarly, the electromagnetic responses of the QGP can be quantified in terms of the electrical conductivity of the medium. Several works have been devoted to understanding the electrical and thermal transport processes in a magnetized QCD medium [33-40]. Notably, the magnetic field induces anisotropy in the transport processes and, in the strong field limit, the dominant contribution arises from the longitudinal component of the transport coefficients associated with the thermal and electric charge transport processes.

The thermoelectric or Seebeck effect describes phenomena in which a temperature gradient generates an electric 
current in an electrically conducting medium and vice versa. The thermoelectric behavior has been well studied for condensed matter systems [41,42]. In a recent study [43], a related coefficient, namely, the Seebeck coefficient, has been investigated for a dense hadronic medium. The thermoelectric behavior of quark matter has started receiving attention very recently [44]. It is important to note that a finite quark chemical potential is required to obtain a nonzero thermoelectric current in the medium. Further, the presence of the magnetic field leads to anisotropic thermoelectric transport process in the medium. The thermoelectric effect in the presence of the magnetic field can be studied in terms of magnetic-field-dependent Seebeck coefficient and Nernst coefficient in the weakly magnetized QGP medium. Transport coefficients characterizing the thermoelectric behavior of the magnetized QCD medium, Seebeck and Nernst coefficients, has been estimated in both weak field [45,46] and strong field regimes [47]. It would be an interesting aspect to study the thermoelectric responses of the interacting QCD medium while including the effects of collisions and realistic equation of state (EOS) for both the cases of vanishing and nonvanishing magnetic fields. This sets the motivation for our investigations.

In the current analysis, the thermoelectric responses of the interacting two-flavor QGP medium and the associated transport coefficients have been estimated within an effective quasiparticle model. The gluonic, quark, and antiquark degrees of freedom are modeled by utilizing the effective fugacity quasiparticle model (EQPM) [48,49]. Knowledge about the system away from equilibrium can be obtained by solving the consistently developed effective Boltzmann equation within the EQPM [50]. The collisional aspects of the interacting medium are incorporated in the analysis through relaxation time approximation (RTA) and Bhatnagar-Gross-Krook (BGK) collision kernels. The RTA turns out to be a viable approach to explore the dissipative process and the associated transport coefficients of the QCD medium [51-54]. In a recent study [55], the authors have derived dissipative hydrodynamics employing the RTA while including the transitions between quarks and gluons (inelastic interactions such as pair production and annihilation processes). The BGK kernel is the improvement over the RTA such that it conserves the particle number exactly. The formalism of the thermoelectric responses has been extended to a magnetized nuclear matter in terms of the magnetic-field-dependent Seebeck coefficient and Hall-type Nernst coefficient. In a recent work [56], we have studied the relative significance of electric charge transport in the presence of an external electric field and thermal transport in a weakly magnetized medium in terms of the Wiedemann-Franz law. The present analysis is to understand the physics of a generated electric field due to the temperature gradient in the collisional hot QCD medium. The dependence of the thermoelectric effect on ambient temperature and magnetic fields and other parameters of a collision, such as quark chemical potential, are studied in the presence of a realistic EOS.

The manuscript is organized as follows. The mathematical formulation of thermoelectric transport in a collisional hot QCD medium within the EQPM effective kinetic theory is presented in Sec. II. Section III describes the thermoelectric behavior and the associated transport coefficients of a magnetized QGP. Section IV is devoted to the results and followed by discussion. Finally, in Sec. V, the present analysis is summarized with an outlook.

Notations and conventions. - The fractional charge of the up and down quarks are $q_{f}=2 e / 3$ and $-e / 3$, respectively. We define $u^{\mu}$ as the normalized unit vector with $u^{\mu} u_{\mu}=1$ and $\Delta^{\mu \nu} \equiv g^{\mu \nu}-u^{\mu} u^{\nu}$ as the projection operator orthogonal to $u^{\mu}$ where $g^{\mu \nu}=\operatorname{diag}(1,-1,-1,-1)$ is the metric tensor. The index $k$ represents the particle species. In the absence of a magnetic field, the quantity $g_{k}$ denotes the degeneracy factor with $g_{q, \bar{q}}=\sum_{f} 2 N_{c}$, where $\sum_{f}$ is the sum over flavor $f$. We consider $N_{f}=2$ and massless limit of quarks in the present analysis.

\section{EFFECTIVE DESCRIPTION OF THERMOELECTRIC EFFECT IN THERMAL QCD MEDIUM}

The diffusion of the charge carriers due to the temperature gradient in a medium results in the generation of the electric field, and the thermoelectric behavior of the medium can be quantified with the associated transport parameter, the Seebeck coefficient. The effective description of the thermoelectric behavior of the collisional QCD medium requires knowledge of the nonequilibrium part of the distribution function along with the proper modeling of the system at equilibrium. To that end, we employ the EQPM description of the medium. The model initiated with the ansatz that the realistic EOS can be interpreted in terms of noninteracting quasiparticles with temperature-dependent effective fugacities. The particle four flow $N^{\mu}$ is described within the EQPM as follows [50]:

$$
\begin{aligned}
N^{\mu}= & g_{q} \int \frac{d^{3}\left|\tilde{\mathbf{p}}_{q}\right|}{(2 \pi)^{3} \omega_{q}} p_{q}^{\mu}\left(f_{q}\left(x, \tilde{p}_{q}\right)-f_{\bar{q}}\left(x, \tilde{p}_{\bar{q}}\right)\right) \\
& +\delta \omega_{q} g_{q} \int \frac{d^{3}\left|\tilde{\mathbf{p}}_{q}\right|}{(2 \pi)^{3} \omega_{q}} \frac{\left\langle\tilde{p}_{q}^{\mu}\right\rangle}{\epsilon_{q}}\left(f_{q}\left(x, \tilde{p}_{q}\right)-f_{\bar{q}}\left(x, \tilde{p}_{\bar{q}}\right)\right),
\end{aligned}
$$

where $\left\langle\tilde{p}_{q}^{\mu}\right\rangle \equiv \Delta_{\nu}^{\mu} \tilde{p}_{q}^{\nu}$ and $\tilde{p}_{q}^{\mu}=\left(\omega_{q}, \tilde{\mathbf{p}}_{\mathbf{q}}\right)$ is the dressed fourmomentum of the quasiquark in the medium. For the system not very far from local equilibrium, the EQPM momentum distribution function is defined as

$$
f_{k}=f_{k}^{0}+\delta f_{k}, \quad f_{k}^{0}=\frac{z_{k} \exp \left[-\beta\left(u \cdot p_{k}-a_{k} \mu\right)\right]}{1 \pm z_{k} \exp \left[-\beta\left(u \cdot p_{k}-a_{k} \mu\right)\right]},
$$


with $\left|\delta f_{k}\right| \ll f_{k}^{0}, \mu$ as the quark chemical potential, and $a_{k}=1,-1$, and 0 for quarks, antiquarks, and gluons, respectively. Here, $f_{k}^{0}$ is the EQPM equilibrium distribution function and $\delta f_{k}$ nonequilibrium part of the momentum distribution. The parameter $z_{k}$ is the effective fugacity that encodes the medium interactions through lattice EOS. The quasiparticle four-momenta are related to the bare particle momenta $p_{k}^{\mu}=\left(\epsilon_{k}, \mathbf{p}_{k}\right)$ through $z_{k}$. The physical significance of the fugacity parameter can be understood from the energy dispersion relation as

$$
\tilde{p}_{k}{ }^{\mu}=p_{k}^{\mu}+\delta \omega_{k} u^{\mu}, \quad \delta \omega_{k}=T^{2} \partial_{T} \ln \left(z_{k}\right),
$$

such that the quasiparticle energy $\omega_{k}=\epsilon_{k}+\delta \omega_{k}$, where $\epsilon_{k}=\left|\tilde{\mathbf{p}}_{k}\right|$. Since $z_{k}$ are not associated with any conserved current in the medium the temperature behavior of quark and antiquark effective fugacities remains the same, i.e., $z_{q}=z_{\bar{q}}$. The effective fugacity model was seen to be thermodynamically consistent without adding any external parameter realizing the medium as a grand canonical ensemble of quarks or antiquarks and gluons. The effective grand canonical partition function for the hot QCD medium which yields the forms of the momentum distribution function takes the form [48]

$$
Z_{\text {eff }}=Z_{g} Z_{q} Z_{\bar{q}}
$$

where the quark or antiquark and gluonic contribution, respectively, take the forms as follows:

$\ln Z_{k}= \pm g_{k} V \int \frac{d\left|\mathbf{p}_{k}\right|}{(2 \pi)^{3}} \ln \left(1 \pm z_{k} \exp \left(-\beta\left(\epsilon_{k}-a_{k} \mu\right)\right)\right)$,

where $V$ is the volume, - is for the gluonic and + is for the quark sector, respectively. Employing the thermodynamic definition of pressure $P=P_{g}+P_{q}+P_{\bar{q}}$ for a grand canonical ensemble, $P \beta V=\ln Z_{\text {eff }}$, we can fix the fugacity parameters in the model. The temperature dependence of effective fugacity for gluons, quarks and antiquarks can be estimated by fitting the pressure obtained within the EQPM description with the lattice QCD results. The gluonic fugacity parameter is determined from the contribution to the lattice pressure of the pure gluonic sector [pure $\mathrm{SU}(3)$ gauge theory]. Similarly, $z_{q}$ captures the interactions in the quark sector and can be fitted with the QCD lattice EOS. The parametric forms for $z_{q}$ and $z_{g}$ with fitting parameters and the temperature behavior of the fugacities are well investigated in Ref. [48]. The net baryon density $n$ can be defined from the zeroth component of the $N^{\mu}$ and takes the following form in the massless limit:

$$
n=\frac{T^{3}}{\pi^{2}} \sum_{k} g_{k} a_{k} \text { PolyLog }\left[3,-z_{q} e^{-\alpha_{k}}\right],
$$

where $\alpha_{k}=\beta a_{k} \mu$. Similarly, the microscopic definition of current density in the QCD medium within the EQPM has the form as follows:

$j^{i}=\sum_{k} g_{k} \int d \tilde{P}_{k} q_{f_{k}} v_{k}^{i} \delta f_{k}-\sum_{k} \delta \omega_{k} g_{k} \int d \tilde{P}_{k} q_{f_{k}} \frac{v_{k}^{i}}{\epsilon_{k}} \delta f_{k}$,

with $d \tilde{P}_{k}=\frac{d^{3}\left|\tilde{\mathbf{p}}_{k}\right|}{(2 \pi)^{3}}$ and $v^{i}=\frac{\tilde{p}^{i}}{\tilde{p}^{0}}$. The system away from equilibrium $\delta f_{k}$ can be described by the relativistic Boltzmann equation. The effective Boltzmann within the EQPM takes the following form:

$\tilde{p}_{k}^{\mu} \partial_{\mu} f_{k}\left(x, \tilde{p}_{k}\right)+\left(F_{k}^{\mu}\left(u \cdot \tilde{p}_{k}\right)+q_{f_{k}} F^{\mu \nu} \tilde{p}_{k \nu}\right) \partial_{\mu}^{\left(\tilde{p}_{k}\right)} f_{k}=C\left[f_{k}\right]$,

where $C\left[f_{k}\right]$ is the collision kernel that quantifies the rate of change of distribution functions due to the collisional processes in the medium. Here, $F^{\mu \nu}$ represents the electromagnetic field strength tensor and $F_{k}^{\mu}=-\partial_{\nu}\left(\delta \omega_{k} u^{\nu} u^{\mu}\right)$ denotes the mean field force term that arises from the basic conservation laws in the medium. We solve the Boltzmann equation (8) to obtain $\delta f_{k}$ with a proper choice of the collision kernel.

\section{A. Seebeck coefficient within RTA}

Within the RTA, the physics of collisions in a QCD medium is approximated in terms of the thermal relaxation time $\tau_{R_{k}}$ as follows:

$$
C_{k}=-\left(u \cdot \tilde{p}_{k}\right) \frac{\delta f_{k}}{\tau_{R_{k}}} .
$$

The relaxation time for the $2 \rightarrow 2$ elastic scattering processes in the medium has been included in the present analysis [57]. Notably, in a strongly magnetized medium $1 \rightarrow 2$ processes such as quark-antiquark annihilation and vice versa are kinematically possible and considered as the dominant processes in the medium [58]. In the massless case, the relaxation time for quarks and antiquarks takes the following form [36,59]:

$$
\tau_{R}=\frac{1}{5.1 T \alpha_{\mathrm{eff}}^{2} \ln \left(\frac{1}{\alpha_{\mathrm{eff}}}\right)\left(1+0.12\left(2 N_{f}+1\right)\right)},
$$

where $\alpha_{\text {eff }}$ is the effective coupling that can be realized as the charge renormalization within the EQPM description and is related to running coupling constant $\alpha_{s}(T, \mu)$ as

$$
\begin{aligned}
\frac{\alpha_{\mathrm{eff}}}{\alpha_{s}(T, \mu)}= & {\left[\frac{2 N_{c}}{\pi^{2}} \operatorname{PolyLog}\left[2, z_{g}\right]-\frac{2 N_{f}}{\pi^{2}} \operatorname{PolyLog}\left[2,-z_{q}\right]\right.} \\
& \left.+\mu^{2} \frac{N_{f}}{\pi^{2}} \frac{z_{q}}{1+z_{q}}\right] \frac{1}{\left(\frac{N_{c}}{3}+\frac{N_{f}}{6}+\mu^{2} \frac{N_{f}}{2 \pi^{2}}\right)}
\end{aligned}
$$


It is important to emphasize that the realistic EOSs are embedded in the relaxation time through $\alpha_{\text {eff }}$. Substituting Eq. (9) in Eq. (8) and employing an iterative ChapmanEnskog-like approach [60], we solve the Boltzmann equation while considering the force term as $\mathbf{F}=q_{f_{k}} \mathbf{E}$ in the case of a finite electric field. The first-order correction to the distribution can be expressed as

$$
\begin{aligned}
\delta f_{k}= & \tau_{R_{k}}\left[\frac{1}{T}\left\{\tilde{p}_{k}^{0} \partial_{0} T+\tilde{p}_{k}^{i} \partial_{i} T\right\}+\frac{T}{\tilde{p}_{k}^{0}}\left\{\tilde{p}_{k}^{0} \partial_{0} \alpha_{k}\right.\right. \\
& \left.+\tilde{p}_{k}^{i} \partial_{i} \alpha_{k}\right\}-\frac{1}{\tilde{p}_{k}^{0}}\left\{\tilde{p}_{k}^{0} \tilde{p}_{k}^{\nu} \partial_{0} u_{\nu}+\tilde{p}_{k}^{i} \tilde{p}_{k}^{\nu} \partial_{i} u_{\nu}\right\} \\
& \left.+\theta \delta \omega_{k}-q_{f_{k}} \overline{\mathbf{v}}_{k} \cdot \mathbf{E}\right] \frac{\partial f_{k}^{0}}{\partial \epsilon_{k}},
\end{aligned}
$$

where $\overline{\mathbf{v}}_{k}=\mathbf{v}_{k} \frac{\omega_{k}}{\epsilon_{k}}$. Here, $\theta=\partial_{\mu} u^{\mu}$ and the traceless part of the velocity gradient $\left\langle\left\langle\partial_{\mu} u_{\nu}\right\rangle\right\rangle$ acts as the source terms for the bulk and shear viscous force in the medium. Simplifying Eq. (12) by Gibbs-Duhem relation $\partial_{i}\left(\frac{\mu}{T}\right)=$ $-\frac{h}{T^{2}}\left(\partial_{i} T-\frac{T}{n h} \partial_{i} P\right)$, where $h=\frac{\varepsilon+P}{n}, \varepsilon$ and $P$ are the enthalpy, energy density and pressure of the system, respectively, we obtain

$$
\delta f_{k}=\tau_{R_{k}} \frac{\partial f_{k}^{0}}{\partial \epsilon_{k}}\left[\left(\omega_{k}-a_{k} h\right) v_{k}^{i} \frac{\partial_{i} T}{T}-q_{f_{k}} \bar{v}_{k}^{i} E^{i}\right] .
$$

It is important to emphasize that in the steady state the momentum conservation gives $\partial_{i} P=0$. Equation (13) denotes the nonequilibrium correction to the distribution function due to the temperature gradient and electric field in the medium. Substituting Eq. (13) in the electric current density as described in Eq. (7), we obtain

$$
\begin{aligned}
\mathbf{j}= & \sum_{k} \frac{g_{k} \tau_{R_{k}} q_{f_{k}}}{3 T} \int d \tilde{P}_{k} \frac{\left|\tilde{\mathbf{p}}_{k}\right|^{2}}{\omega_{k}^{2}}\left(\omega_{k}-a_{k} h\right) \frac{\partial f_{k}^{0}}{\partial \epsilon_{k}} \nabla T \\
& -\sum_{k} \delta \omega_{k} \frac{g_{k} \tau_{R_{k}} q_{f_{k}}}{3 T} \int d \tilde{P}_{k} \frac{\left|\tilde{\mathbf{p}}_{k}\right|}{\omega_{k}^{2}}\left(\omega_{k}-a_{k} h\right) \frac{\partial f_{k}^{0}}{\partial \epsilon_{k}} \nabla T \\
& -\sum_{k} \frac{g_{k} \tau_{R_{k}} q_{f_{k}}^{2}}{3} \int d \tilde{P}_{k} \frac{\left|\tilde{\mathbf{p}}_{k}\right|^{2}}{\omega_{k}} \frac{1}{\epsilon_{k}} \frac{\partial f_{k}^{0}}{\partial \epsilon_{k}} \mathbf{E} \\
& +\sum_{k} \frac{g_{k} \tau_{R_{k}} q_{f_{k}}^{2}}{3} \int d \tilde{P}_{k} \frac{\left|\tilde{\mathbf{p}}_{k}\right|^{2}}{\omega_{k}} \frac{1}{\epsilon_{k}} \frac{\partial f_{k}^{0}}{\partial \epsilon_{k}} \mathbf{E} .
\end{aligned}
$$

The thermoelectric effect can be described by setting up $\mathbf{j}=0$ in a steady state, and hence we have

$$
\mathbf{E}=S \boldsymbol{\nabla} T,
$$

such that the generated electric field is proportional to the temperature gradient which can be quantified in terms of the Seebeck coefficient $S$. Employing Eqs. (14) and (15) and performing the thermodynamic integrals within the
EQPM description, the Seebeck coefficient takes the form as follows:

$$
S=\frac{I_{1}(T, \mu)}{I_{2}(T, \mu)}
$$

where $I_{1}$ and $I_{2}$ can be defined in terms of PolyLog functions as, respectively,

$$
\begin{aligned}
I_{1}= & -\frac{1}{6 \pi^{2}} \sum_{k} g_{k} \tau_{R_{k}} q_{f_{k}}\left[-6 T^{2} \operatorname{PolyLog}\left[3,-e^{\alpha_{k}} z_{k}\right]\right. \\
+ & 4 \delta \omega_{k} T \operatorname{PolyLog}\left[2,-e^{\alpha_{k}} z_{k}\right] \\
& +a_{k} h T\left(2 \operatorname{PolyLog}\left[2,-e^{\alpha_{k}} z_{k}\right]\right. \\
& \left.\left.+3 \frac{\delta \omega_{k}}{T} \log \left[1+e^{\alpha_{k}} z_{k}\right]\right)\right] \\
I_{2}= & \frac{1}{3 \pi^{2}} \sum_{k} g_{k} \tau_{R_{k}} q_{f_{k}}^{2}\left[T^{2} \operatorname{PolyLog}\left[2,-e^{\alpha_{k}} z_{k}\right]\right. \\
& \left.+\delta \omega_{k} T \log \left[1+e^{\alpha_{k}} z_{k}\right]\right] .
\end{aligned}
$$

It is important to note that the term $I_{2}$ is related to the electrical conductivity of the QGP medium, i.e., $I_{2}=-\sigma_{e}$ in the case of a vanishing magnetic field. The temperature behavior of the $\sigma_{e}$ and the Seebeck coefficient critically depends on the thermal relaxation time. The effect of collisions can be further studied with the BGK collisional term and could be thought of as an improvement over the RTA results.

\section{B. Seebeck coefficient within BGK kernel}

The BGK collisional aspects are observed to have a significant impact on the collective modes, refractive index, and electric charge transport process of the QCD medium [61-65]. The BGK collision term takes the form as follows:

$C\left[f_{k}\right]=-\nu\left[f_{k}-\frac{N_{k}}{N_{k}^{0}} f_{k}^{0}\right]=-\nu\left[\delta f_{k}-\frac{f_{k}^{0}}{N_{k}^{0}} \int d \tilde{P}_{k} \delta f_{k}\right]$,

where $\nu$ is the collisional frequency which is independent of the momentum of particles and

$$
N_{k}=\int d \tilde{P}_{k} f_{k}, \quad N_{k}^{0}=\int d \tilde{P}_{k} f_{k}^{0}
$$

denote the particle densities of the $k$ th species. The BGK collision kernel preserves number conservation instantaneously, unlike conventional RTA integral, i.e., $\int d \tilde{P}_{k} C\left[f_{k}\right]=0$. Note that, in the limit $\frac{N_{k}}{N_{k}^{0}}=1$, the BGK collisional term reduces to RTA kernel. Substituting 
Eq. (19) in Eq. (8) and solving the Boltzmann equation, we have

$$
\begin{aligned}
\delta f_{k}= & \delta f_{k}^{(0)}+i \nu(i D)^{-1} \frac{f_{k}^{0}}{N_{k}^{0}}\left[\int d \tilde{P}_{k}^{\prime} \delta f_{k}^{(0)}\left(\tilde{p}^{\prime}, X\right)\right] \\
& +i \nu(i D)^{-1} \frac{f_{k}^{0}}{N_{k}^{0}} \frac{i \nu}{N_{k}^{0}}\left[\int d \tilde{P}_{k}^{\prime}(i D)^{-1} f_{k}^{0}\right. \\
& \left.\times \int d \tilde{P}_{k}^{\prime \prime} \delta f_{k}^{(0)}\left(\tilde{p}^{\prime \prime}, X\right)\right]+\cdots,
\end{aligned}
$$

where $D=v \cdot \partial_{X}+\nu$ with $v=\left(1, \mathbf{v}_{k}\right)$ and the RTA equivalent form of the part of distribution function away from equilibrium $\delta f_{k}^{(0)}$ takes the following form:

$\delta f_{k}^{(0)}=\nu^{-1} \frac{\tilde{p}_{k}^{i}}{\tilde{p}_{k}^{0}}\left[\left(\omega_{k}-a_{k} h\right) \frac{\partial_{i} T}{T}-\frac{\tilde{p}_{k}^{0}}{\epsilon_{k}} q_{f_{k}} E^{i}\right] \frac{\partial f_{k}^{0}}{\partial \epsilon_{k}}$.

Employing Eq. (21) in Eq. (7) and keeping terms in leading order of $\nu^{-1}$, the current density takes the following form:

$$
\begin{aligned}
j^{i}= & \sum_{k} g_{k} \nu^{-1} q_{f_{k}} \int d \tilde{P}_{k} v_{k}^{i}\left\{\left[\frac{\tilde{p}_{k}^{i}}{\omega_{k}}\left(\omega_{k}-a_{k} h\right) \frac{\partial_{i} T}{T}-\frac{\tilde{p}_{k}^{i}}{\epsilon_{k}} q_{f_{k}} E^{i}\right] \frac{\partial f_{k}^{0}}{\partial \epsilon_{k}}+\frac{f_{k}^{0}}{N_{k}^{0}} \nu^{-1} \int d \tilde{P}_{k}^{\prime}\right. \\
& \left.\times\left[\frac{\tilde{p}_{k}^{\prime i}}{\omega_{k}^{\prime}}\left(\omega_{k}^{\prime}-a_{k} h\right) \frac{\partial_{i} T}{T}-\frac{\tilde{p}_{k}^{\prime i}}{\epsilon_{k}^{\prime}} q_{f_{k}} E^{i}\right] \frac{\partial f_{k}^{0}}{\partial \epsilon_{k}^{\prime}}\right\}+\sum_{k} \delta \omega_{k} g_{k} \nu^{-1} q_{f_{k}} \int d \tilde{P}_{k} \frac{v_{k}^{i}}{\left|\tilde{\mathbf{p}}_{k}\right|}\left\{\left[\frac{\tilde{p}_{k}^{i}}{\omega_{k}}\left(\omega_{k}-a_{k} h\right) \frac{\partial_{i} T}{T}\right.\right. \\
& \left.\left.-\frac{\tilde{p}_{k}^{i}}{\epsilon_{k}} q_{f_{k}} E^{i}\right] \frac{\partial f_{k}^{0}}{\partial \epsilon_{k}}+\frac{f_{k}^{0}}{N_{k}^{0}} \nu^{-1} \int d \tilde{P}_{k}^{\prime}\left[\frac{\tilde{p}_{k}^{\prime i}}{\omega_{k}^{\prime}}\left(\omega_{k}^{\prime}-a_{k} h\right) \frac{\partial_{i} T}{T}-\frac{\tilde{p}_{k}^{\prime i}}{\epsilon_{k}^{\prime}} q_{f_{k}} E^{i}\right] \frac{\partial f_{k}^{0}}{\partial \epsilon_{k}^{\prime}}\right\} .
\end{aligned}
$$

Setting up $\mathbf{j}=0$ by employing Eq. (23) and performing the thermodynamic integrals, the Seebeck coefficient within the BGK collision kernel can be defined as

$$
\begin{aligned}
& S=\frac{K_{1}(T, \mu)}{K_{2}(T, \mu)} \\
& K_{1}=-\frac{1}{6 \pi^{2}} \sum_{k} \frac{g_{k} q_{f_{k}}}{\nu}\left[-6 T^{2} \operatorname{PolyLog}\left[3,-e^{\alpha_{k}} z_{k}\right]\right. \\
&+4 \delta \omega_{k} T \operatorname{PolyLog}\left[2,-e^{\alpha_{k}} z_{k}\right]+a_{k} h T \\
& \times\left(2 \operatorname{PolyLog}\left[2,-e^{\alpha_{k}} z_{k}\right]+3 \frac{\delta \omega_{k}}{T} \log \left[1+e^{\alpha_{k}} z_{k}\right]\right) \\
&-2 \frac{A_{k}}{N_{k}^{0}}\left(-T^{2} \operatorname{PolyLog}\left[3,-e^{\alpha_{k}} z_{k}\right]\right. \\
&+\left.\left.\delta \omega_{k} T \operatorname{PolyLog}\left[2,-e^{\alpha_{k}} z_{k}\right]\right)\right] \\
& K_{2}= \frac{1}{3 \pi^{2}} \sum_{k} \frac{g_{k} q_{f_{k}}^{2}}{\nu}\left[\left(T^{2} \operatorname{PolyLog}\left[2,-e^{\alpha_{k}} z_{k}\right]\right.\right. \\
&\left.+\delta \omega_{k} T \log \left[1+e^{\alpha_{k}} z_{k}\right]\right) \\
&+\frac{B_{k}}{N_{k}^{0}}\left(-T^{2} \operatorname{PolyL} \log \left[3,-e^{\alpha_{k}} z_{k}\right]\right. \\
&\left.\left.+\delta \omega_{k} T \operatorname{PolyLog}\left[2,-e^{\alpha_{k}} z_{k}\right]\right)\right]
\end{aligned}
$$

where $A_{k}$ and $B_{k}$, respectively, take the forms as follows:

$$
\begin{gathered}
A_{k}=\frac{1}{2 \pi^{2}}\left[6 T^{3} \text { PolyLog }\left[3,-e^{\alpha_{k}} z_{k}\right]-a_{k} h T^{2}\right. \\
\left.\times\left(2 \text { PolyLog }\left[2,-e^{\alpha_{k}} z_{k}\right]+\frac{\delta \omega_{k}}{T} \log \left[1+e^{\alpha_{k}} z_{k}\right]\right)\right], \\
B_{k}=\frac{T^{3}}{\pi^{2}} \operatorname{PolyLog}\left[2,-e^{\alpha_{k}} z_{k}\right] .
\end{gathered}
$$

It is important to note that, in the limit of $z_{k}=1$, the analysis reduces back to that in the case of ultrarelativistic gas of quarks or antiquarks and gluons. The term $\left|K_{2}\right|$ denotes the electrical conductivity of the QGP within the BGK collision kernel. Let us now proceed to discuss the thermoelectric effect in a magnetized QCD medium.

\section{THERMOELECTRIC RESPONSES OF A MAGNETIZED QGP WITHIN THE EQPM}

In a weakly magnetized medium, the temperature is the dominant energy scale in comparison with the strength of the magnetic field. Hence, the energy dispersion of quarks and antiquarks remains intact in the presence of the weak magnetic field, unlike the Landau level dispersion of charged fermions in a strongly magnetized medium. In the presence of a weak magnetic field, the Boltzmann equation has the form

$$
\begin{aligned}
& {\left[-\frac{\partial f_{k}^{0}}{\partial \epsilon_{k}}\left(\omega_{k}-a_{k} h\right) \frac{1}{T}\left(\mathbf{v}_{k} \cdot \nabla T\right)\right]} \\
& +q_{f_{k}}\left[\mathbf{E}+\left(\mathbf{v}_{k} \times \mathbf{B}\right)\right] \cdot \frac{\partial f_{k}}{\partial \tilde{\mathbf{p}}_{k}}=-\frac{\delta f_{k}}{\tau_{R_{k}}} .
\end{aligned}
$$

We consider that the dependence of the magnetic field on the thermal relaxation time for the binary scattering process is 
entering through the one-loop magnetic-field-dependent coupling constant $\alpha_{s}\left(q_{f} B, T\right)$. The form of $\alpha_{s}\left(q_{f} B, T\right)$ for a weakly magnetized medium is presented in Refs. $[21,66]$. A much deeper analysis is needed to obtain the exact form of thermal relaxation to incorporate the effects of the magnetic field and elastic-inelastic interactions in the medium, which is beyond the scope of the present analysis. In the presence of a strong magnetic field, the relaxation time critically depends on the strength of the magnetic field as the $1 \rightarrow 2$ processes are kinematically possible in the medium [58]. The nonequilibrium part of the quasiparton distribution in the presence of a weak magnetic can be obtained by solving the Boltzmann equation. To that end, we choose the following ansatz:

$$
\delta f_{k}=\left(\tilde{\mathbf{p}}_{k} \cdot \boldsymbol{\Xi}\right) \frac{\partial f_{k}^{0}}{\partial \epsilon_{k}}
$$

where $\boldsymbol{\Xi}$ is defined as

$$
\begin{aligned}
\boldsymbol{\Xi}= & \alpha_{1} \mathbf{E}+\alpha_{2} \mathbf{b}+\alpha_{3}(\mathbf{E} \times \mathbf{b})+\alpha_{4} \mathbf{\nabla} T \\
& +\alpha_{5}(\boldsymbol{\nabla} T \times \mathbf{b})+\alpha_{6}(\boldsymbol{\nabla} T \times \mathbf{E}),
\end{aligned}
$$

with unit vector $\mathbf{b}=\frac{\mathbf{B}}{|\mathbf{B}|}$ representing the direction of the magnetic field. In general, there will be other independent force terms other than the thermal driving force and Lorentz force in the Boltzmann equation that corresponds to the momentum transport and the associated viscous coefficients in the medium. Different components of the transport parameters associated with the electric charge, thermal and momentum transport processes in the magnetized medium are studied in Ref. [67]. Since the Lorenz force vanishes in the equilibrium case as $\frac{\partial f_{k}^{0}}{\partial \tilde{\mathbf{p}}_{k}} \propto \mathbf{v}_{k}$, Eq. (29) reduces to the following form:

$$
\begin{gathered}
{\left[-\left(\omega_{k}-a_{k} h\right) \frac{1}{T}\left(\mathbf{v}_{k} \cdot \nabla T\right)+q_{f_{k}}\left(\overline{\mathbf{v}}_{k} \cdot \mathbf{E}\right)\right] \frac{\partial f_{k}^{0}}{\partial \epsilon_{k}}} \\
+q_{f_{k}}\left[\left(\mathbf{v}_{k} \times \mathbf{B}\right) \cdot \boldsymbol{\Xi}\right] \frac{\partial f_{k}^{0}}{\partial \epsilon_{k}}=-\frac{1}{\tau_{R_{k}}}\left(\tilde{\mathbf{p}}_{k} \cdot \boldsymbol{\Xi}\right) \frac{\partial f_{k}^{0}}{\partial \epsilon_{k}} .
\end{gathered}
$$

The unknown parameters $\alpha_{i}(i=1,2, \ldots, 6)$ in the nonequilibrium part of the distribution can be obtained by substituting Eq. (30) in Eq. (32). Hence, we obtain

$$
\begin{aligned}
- & \left(\omega_{k}-a_{k} h\right) \frac{1}{T}\left(\mathbf{v}_{k} \cdot \boldsymbol{\nabla} T\right)+q_{f_{k}}\left(\overline{\mathbf{v}}_{k} \cdot \mathbf{E}\right)+q_{f_{k}} \alpha_{1}(\mathbf{v} \times \mathbf{B}) \cdot \mathbf{E}+q_{f_{k}} \alpha_{2}(\mathbf{v} \times \mathbf{B}) \cdot \mathbf{b}+q_{f_{k}} \alpha_{3}(\mathbf{v} \times \mathbf{B}) \cdot(\mathbf{E} \times \mathbf{b}) \\
& +q_{f_{k}} \alpha_{4}(\mathbf{v} \times \mathbf{B}) \cdot \boldsymbol{\nabla} T+q_{f_{k}} \alpha_{5}(\mathbf{v} \times \mathbf{B}) \cdot(\boldsymbol{\nabla} T \times \mathbf{b})+q_{f_{k}} \alpha_{6}(\mathbf{v} \times \mathbf{B}) \cdot(\boldsymbol{\nabla} T \times \mathbf{E}) \\
= & -\frac{\omega_{k}}{\tau_{R_{k}}}\left[\alpha_{1} \mathbf{v}_{k} \cdot \mathbf{E}+\alpha_{2} \mathbf{v}_{k} \cdot \mathbf{b}+\alpha_{3} \mathbf{v}_{k} \cdot(\mathbf{E} \times \mathbf{b})+\alpha_{4} \mathbf{v}_{k} \cdot \boldsymbol{\nabla} T+\alpha_{5} \mathbf{v}_{k} \cdot(\nabla T \times \mathbf{b})+\alpha_{6} \mathbf{v}_{k} \cdot(\boldsymbol{\nabla} T \times \mathbf{E})\right] .
\end{aligned}
$$

Comparing different independent tensor structures on both sides of Eq. (33), we obtain the following relations for the parameters $\alpha_{i}(i=1,2, \ldots, 6)$ :

$$
\begin{gathered}
-\frac{\omega_{k}}{\tau_{R_{k}}} \alpha_{1}=\frac{\omega_{k}}{\epsilon_{k}} q_{f_{k}}+\alpha_{3} q_{f_{k}}|\mathbf{B}|, \\
-\frac{\omega_{k}}{\tau_{R_{k}}} \alpha_{2}=-\alpha_{3} q_{f_{k}}(\mathbf{B} \cdot \mathbf{E})-\alpha_{5} q_{f_{k}}(\mathbf{B} \cdot \boldsymbol{\nabla} T), \\
-\frac{\omega_{k}}{\tau_{R_{k}}} \alpha_{3}=-\alpha_{1} q_{f_{k}}|\mathbf{B}|, \\
-\frac{\omega_{k}}{\tau_{R_{k}}} \alpha_{4}=-\frac{\left(\omega_{k}-a_{k} h\right)}{T}+\alpha_{5} q_{f_{k}}|\mathbf{B}|, \\
-\frac{\omega_{k}}{\tau_{R_{k}}} \alpha_{5}=-\alpha_{4} q_{f_{k}}|\mathbf{B}|,
\end{gathered}
$$

with $\alpha_{6}=0$. For the quantitative analysis of the thermoelectric effect in a magnetized medium, we consider the magnetic field direction along the $z$ axis and the directions of the electric and temperature gradient in the $x-y$ plane in the present analysis, i.e., $(\mathbf{b} \cdot \mathbf{E})=(\mathbf{b} \cdot \nabla T)=0$. Further solving Eqs. (34)-(38), the parameters take the forms as follows:

$$
\begin{gathered}
\alpha_{1}=-\frac{\tau_{R}}{\epsilon_{k}} \frac{q_{f_{k}}}{\left(1+\tau_{R_{k}}^{2} \Omega_{c k}^{2}\right)}, \\
\alpha_{2}=0, \\
\alpha_{3}=-\frac{\tau_{R_{k}}^{2}}{\epsilon_{k}} \frac{q_{f_{k}}}{\left(1+\tau_{R_{k}}^{2} \Omega_{c k}^{2}\right)} \Omega_{c k}, \\
\alpha_{4}=\frac{\tau_{R_{k}}}{\omega_{k}} \frac{1}{T} \frac{\left(\omega_{k}-a_{k} h\right)}{\left(1+\tau_{R_{k}}^{2} \Omega_{c k}^{2}\right)}, \\
\alpha_{5}=\frac{\tau_{R_{k}}^{2}}{\omega_{k}} \frac{1}{T} \frac{\left(\omega_{k}-a_{k} h\right)}{\left(1+\tau_{R_{k}}^{2} \Omega_{c k}^{2}\right)} \Omega_{c k},
\end{gathered}
$$

where $\Omega_{c k}=\frac{q_{f_{k}}|B|}{\omega_{k}}$ denotes the cyclotron frequency in the presence of the magnetic field. Employing Eqs. (39)-(43) in Eq. (30) we obtain

$$
\begin{aligned}
\delta f_{k}= & {\left[-q_{f_{k}}\left(\overline{\mathbf{v}}_{k} \cdot \mathbf{E}+\tau_{R_{k}} \Omega_{c k} \mathbf{v}_{k} \cdot(\mathbf{E} \times \mathbf{b})\right)\right.} \\
& \left.+\left(\omega_{k}-h_{k}\right) \frac{1}{T}\left(\mathbf{v}_{k} \cdot \nabla T+\tau_{R_{k}} \Omega_{c k} \mathbf{v}_{k} \cdot(\nabla T \times \mathbf{b})\right)\right] \\
& \times \frac{\tau_{R_{k}}}{\left(1+\tau_{R_{k}}^{2} \Omega_{c k}^{2}\right)} \frac{\partial f_{k}^{0}}{\partial \epsilon_{k}} .
\end{aligned}
$$


By substituting Eq. (44) in Eq. (7), the component of the current density along the $x$ axis can be obtained as follows:

$$
\begin{aligned}
j_{x}= & \sum_{k} \frac{g_{k} \tau_{R_{k}} q_{f_{k}}}{3} \int d \tilde{P}_{k} \frac{v_{k}^{2}}{\left(1+\tau_{R_{k}}^{2} \Omega_{c k}^{2}\right)}\left[-\frac{\omega_{k}}{\epsilon_{k}} q_{f_{k}}\left(E_{x}\right.\right. \\
& \left.\left.+\tau_{R_{k}} \Omega_{c k} E_{y}\right)+\left(\omega_{k}-a_{k} h\right) \frac{1}{T}\left(\frac{d T}{d x}+\tau_{R_{k}} \Omega_{c k} \frac{d T}{d y}\right)\right] \frac{\partial f_{k}^{0}}{\partial \epsilon_{k}} \\
& -\sum_{k} \delta \omega_{k} \frac{g_{k} \tau_{R_{k}} q_{f_{k}}}{3} \int d \tilde{P}_{k} \frac{v_{k}^{2}}{\epsilon_{k}\left(1+\tau_{R_{k}}^{2} \Omega_{c k}^{2}\right)}\left[-q_{f_{k}}\left(E_{x}\right.\right. \\
& \left.\left.+\tau_{R_{k}} \Omega_{c k} E_{y}\right)+\left(\omega_{k}-a_{k} h\right) \frac{1}{T}\left(\frac{d T}{d x}+\tau_{R_{k}} \Omega_{c k} \frac{d T}{d y}\right)\right] \frac{\partial f_{k}^{0}}{\partial \epsilon_{k}} .
\end{aligned}
$$

Similarly, the component of current density along the $y$ axis takes the following form:

$$
\begin{aligned}
j_{y}= & \sum_{k} \frac{g_{k} \tau_{R_{k}} q_{f_{k}}}{3} \int d \tilde{P}_{k} \frac{v_{k}^{2}}{\left(1+\tau_{R_{k}}^{2} \Omega_{c k}^{2}\right)}\left[-\frac{\omega_{k}}{\epsilon_{k}} q_{f_{k}}\left(E_{y}\right.\right. \\
& \left.\left.-\tau_{R_{k}} \Omega_{c k} E_{x}\right)+\left(\omega_{k}-a_{k} h\right) \frac{1}{T}\left(\frac{d T}{d y}-\tau_{R_{k}} \Omega_{c k} \frac{d T}{d x}\right)\right] \frac{\partial f_{k}^{0}}{\partial \epsilon_{k}} \\
& -\sum_{k} \delta \omega_{k} \frac{g_{k} \tau_{R_{k}} q_{f_{k}}}{3} \int d \tilde{P}_{k} \frac{v_{k}^{2}}{\epsilon_{k}\left(1+\tau_{R_{k}}^{2} \Omega_{c k}^{2}\right)}\left[-q_{f_{k}}\left(E_{y}\right.\right. \\
& \left.\left.-\tau_{R_{k}} \Omega_{c k} E_{x}\right)+\left(\omega_{k}-a_{k} h\right) \frac{1}{T}\left(\frac{d T}{d y}-\tau_{R_{k}} \Omega_{c k} \frac{d T}{d x}\right)\right] \frac{\partial f_{k}^{0}}{\partial \epsilon_{k}} .
\end{aligned}
$$

The charge carriers traveling along the temperature gradient $(x-y$ plane) generate an electric field that induces an electric current in the opposite direction, and in the steady state the net current vanishes in the medium. By setting up $j_{x}=0$ and $j_{y}=0$, we can represent the generated electric field in terms of the temperature gradient. Hence, from Eqs. (45) and (46) we have

$$
\begin{aligned}
& E_{x}=S_{B} \frac{d T}{d x}+N|\mathbf{B}| \frac{d T}{d y}, \\
& E_{y}=S_{B} \frac{d T}{d y}-N|\mathbf{B}| \frac{d T}{d x},
\end{aligned}
$$

where $S_{B}$ and $N$ are the Seebeck and Nernst coefficients, respectively, associated with the thermoelectric transport process in the presence of the magnetic field. In the case of a vanishing magnetic field, Eqs. (47) and (48) reduce to Eq. (15) such that the coefficient $S_{B}$ reduces to $S$ and the Nernst coefficient vanishes in the medium. The $S_{B}$ and $N$ take the following forms:

$$
S_{B}=\frac{L_{1} L_{3}+L_{2} L_{4}}{L_{1}^{2}+L_{2}^{2}}, \quad N|\mathbf{B}|=\frac{L_{1} L_{4}-L_{2} L_{3}}{L_{1}^{2}+L_{2}^{2}},
$$

where $L_{i}(i=1,2,3,4)$ are the thermodynamic integrals in the presence of the magnetic field and can be defined as follows:

$$
\begin{gathered}
L_{1}=\frac{1}{3} \sum_{k} g_{k} \tau_{R_{k}} q_{f_{k}}^{2}\left\{\int d \tilde{P}_{k} \frac{\left|\tilde{\mathbf{p}}_{k}\right|^{2}}{\omega_{k}} \frac{1}{\epsilon_{k}} \frac{1}{\left(1+\tau_{R_{k}}^{2} \Omega_{c k}^{2}\right)} \frac{\partial f_{k}^{0}}{\partial \epsilon_{k}}-\delta \omega_{k} \int d \tilde{P}_{k} \frac{\left|\tilde{\mathbf{p}}_{k}\right|^{2}}{\omega_{k}} \frac{1}{\epsilon_{k}^{2}} \frac{1}{\left(1+\tau_{R_{k}}^{2} \Omega_{c k}^{2}\right)} \frac{\partial f_{k}^{0}}{\partial \epsilon_{k}}\right\}, \\
L_{2}=\frac{1}{3} \sum_{k} g_{k} \tau_{R_{k}} q_{f_{k}}^{2}\left\{\int d \tilde{P}_{k} \frac{\left|\tilde{\mathbf{p}}_{k}\right|^{2}}{\omega_{k}} \frac{1}{\epsilon_{k}} \frac{\tau_{R_{k}} \Omega_{c k}}{\left(1+\tau_{R_{k}}^{2} \Omega_{c k}^{2}\right)} \frac{\partial f_{k}^{0}}{\partial \epsilon_{k}}-\delta \omega_{k} \int d \tilde{P}_{k} \frac{\left|\tilde{\mathbf{p}}_{k}\right|^{2}}{\omega_{k}} \frac{1}{\epsilon_{k}^{2}} \frac{\tau_{R_{k}} \Omega_{c k}}{\left(1+\tau_{R_{k}}^{2} \Omega_{c k}^{2}\right)} \frac{\partial f_{k}^{0}}{\partial \epsilon_{k}}\right\}, \\
L_{3}=\frac{1}{3 T} \sum_{k} g_{k} \tau_{R_{k}} q_{f_{k}}\left\{\int d \tilde{P}_{k} \frac{\left|\tilde{\mathbf{p}}_{k}\right|^{2}}{\omega_{k}^{2}} \frac{\left(\omega_{k}-a_{k} h\right)}{\left(1+\tau_{R_{k}}^{2} \Omega_{c k}^{2}\right)} \frac{\partial f_{k}^{0}}{\partial \epsilon_{k}}-\delta \omega_{k} \int d \tilde{P}_{k} \frac{\left|\tilde{\mathbf{p}}_{k}\right|}{\omega_{k}^{2}} \frac{\left(\omega_{k}-a_{k} h\right)}{\left(1+\tau_{R_{k}}^{2} \Omega_{c k}^{2}\right)} \frac{\partial f_{k}^{0}}{\partial \epsilon_{k}}\right\}, \\
L_{4}=\frac{1}{3 T} \sum_{k} g_{k} \tau_{R_{k}} q_{f_{k}}\left\{\int d \tilde{P}_{k} \frac{\left|\tilde{\mathbf{p}}_{k}\right|^{2}}{\omega_{k}^{2}} \frac{\tau_{R_{k}} \Omega_{c k}\left(\omega_{k}-a_{k} h\right)}{\left(1+\tau_{R_{k}}^{2} \Omega_{c k}^{2}\right)} \frac{\partial f_{k}^{0}}{\partial \epsilon_{k}}-\delta \omega_{k} \int d \tilde{P}_{k} \frac{\left|\tilde{\mathbf{p}}_{k}\right|}{\omega_{k}^{2}} \frac{\tau_{R_{k}} \Omega_{c k}\left(\omega_{k}-a_{k} h\right)}{\left(1+\tau_{R_{k}}^{2} \Omega_{c k}^{2}\right)} \frac{\partial f_{k}^{0}}{\partial \epsilon_{k}}\right\} .
\end{gathered}
$$

The integrals $L_{2}$ and $L_{4}$ vanish at $\mathbf{B}=0$, and hence the Nernst coefficient vanishes in the absence of a magnetic field.

In a strongly magnetized medium, the motion of fermions is constrained in the direction of the magnetic field. Various transport processes in the QCD medium have been initially studied in the lowest Landau level approximation $\left(T^{2} \ll\left|q_{f_{k}} B\right|\right)$ [58] and later on the more realistic regime $g T \ll \sqrt{\left|q_{f} B\right|}$ where higher Landau level contributions are significant [68]. The longitudinal current density $j_{z}$ due to the 1+1-dimensional Landau level dynamics of the charged particles in the presence of the strong magnetic field has been studied in Ref. [69]. Following the same formalism and invoking $j_{z}=0$, the Seebeck coefficient in the strongly magnetized medium $S_{B}^{\|}$can be defined as

$$
\mathbf{E}=S_{B}^{\|} \nabla T, \quad S_{B}^{\|}=\frac{I_{1}^{\|}\left(q_{f} B, T, \mu\right)}{I_{2}^{\|}\left(q_{f} B, T, \mu\right)},
$$

where the integral takes the form 


$$
\begin{aligned}
I_{1}^{\|}= & -\sum_{l=0}^{\infty} \sum_{k} g_{l} \frac{\left|q_{f_{k}} B\right|}{2 \pi} \frac{N_{c}}{T^{2}} \int_{-\infty}^{\infty} \frac{d \tilde{p}_{z_{k}}}{2 \pi} \tau_{\mathrm{eff}} \frac{\left(\omega_{k}^{l}-a_{k} h^{l}\right)}{\omega_{k}^{l 2}} \\
& \times \tilde{p}_{z_{k}}^{2} f_{k}^{l 0}\left(1-f_{k}^{l 0}\right) \\
& +\sum_{l=0}^{\infty} \sum_{k} g_{l} \delta \omega_{k} \frac{\left|q_{f_{k}} B\right|}{2 \pi} \frac{N_{c}}{T^{2}} \int_{-\infty}^{\infty} \frac{d \tilde{p}_{z_{k}}}{2 \pi} \tau_{\mathrm{eff}} \frac{\left(\omega_{k}^{l}-a_{k} h^{l}\right)}{\omega_{k}^{l 2}} \\
& \times \frac{\tilde{p}_{z_{k}}^{2}}{E_{l}} f_{k}^{l 0}\left(1-f_{k}^{l 0}\right) .
\end{aligned}
$$

Here, $g_{l}=\left(2-\delta_{l 0}\right)$ is the spin degeneracy with $l$ as the Landau level, $h^{l}$ is the enthalpy in the strongly magnetized QGP, and $\omega_{k}^{l}=E_{l}+\delta \omega_{k}$ with $E_{l}=\sqrt{p_{z}^{2}+2 l\left|q_{f_{k}} B\right|}$. The EQPM particle distribution function in a strong magnetic field takes the form

$$
f_{k}^{l 0}=\frac{z_{k} \exp \left[-\beta\left(E_{l}-a_{k} \mu\right)\right]}{1+z_{k} \exp \left[-\beta\left(E_{l}-a_{k} \mu\right)\right]} .
$$

Similarly, the integral $I_{2}^{\|}$can be defined as

$$
I_{2}^{\|}=-\sigma_{\|},
$$

where $\sigma_{\|}$is the longitudinal electrical conductivity within the EQPM as described in Ref. [70]. Here, $\tau_{\text {eff }}$ is the thermal relaxation time for dominant $1 \rightarrow 2$ processes in the strongly magnetized medium [58].

\section{RESULTS AND DISCUSSIONS}

We initiate the discussions with the temperature dependence of the Seebeck coefficient in the two-flavor QGP medium. The dependence of quark chemical potential and thermal medium interactions on the temperature behavior of the thermoelectric coefficient is depicted in Fig. 1 (left) in the case of a vanishing magnetic field within the RTA. It is observed that the mean field contributions that arise from the hot medium interactions to the Seebeck coefficient are more pronounced in the temperature regimes not very far from the transition temperature $T_{c}$. In a previous study [56], we have studied the relative behavior of electric and thermal transport in the medium in terms of the Wiedemann-Franz law and observed that the law is violated with the inclusion of EOS effects in temperature regimes near $T_{c}$. In a similar line, we have observed that the hot QCD medium interactions significantly modify the low-temperature behavior of the thermoelectric effect in the medium. The negative sign of $S$ is arising from the term $\left(\omega_{k}-a_{k} h\right)$ for the two-flavor system within the EQPM, in contrast with the term $\left(\omega_{k}-a_{k} h\right)^{2}$ while describing the Lorentz number $L=\frac{\kappa}{\sigma_{e} T}$, where $\kappa$ is the thermal conductivity of the medium. At very high temperature, the EQPM results reduce back to the results of noninteracting medium owing to the fact that $z_{k} \rightarrow 1$ and $\delta \omega_{k} \rightarrow 0$ at the asymptotic limit. At the massless and ideal EOS limits, the coefficient $S$ vanishes for the three-flavor $\mathrm{QGP}$ at $\mathbf{B}=0$. Along with the temperature gradient in the medium, a finite quark chemical potential is also required for the thermoelectric effect in the hot QCD medium [44]. This is attributed to the fact that, in the QGP medium, there are positive and negative charge carriers for the transport process, unlike in the condensed matter system. The quark chemical potential is seen to have a strong dependence on the thermoelectric effect in the medium. The coefficient increases with an increase in the quark chemical potential at a particular temperature. This observation is in line with the results of Ref. [44]. For a large temperature regime, the enthalpy to the net quark number density ratio behaves as $h \sim T \operatorname{coth}(\mu / T)$ and $S \sim-\operatorname{coth}(\mu / T)$ for the massless limit. Within the limit $\frac{\mu}{T} \ll 1$, we have $\operatorname{coth}(\mu / T) \sim T / \mu$ in the leading order. However, it is important to note that, in contrast to the condensed matter systems, a finite quark chemical potential is required for the thermoelectric effect to be observed.

The collisional aspects of the QGP medium are embedded in the analysis through the RTA and BGK kernels. In Fig. 1 (right), the ratio of BGK description of the Seebeck coefficient to the RTA result is plotted as a function of temperature. The BGK description of the Seebeck coefficient is

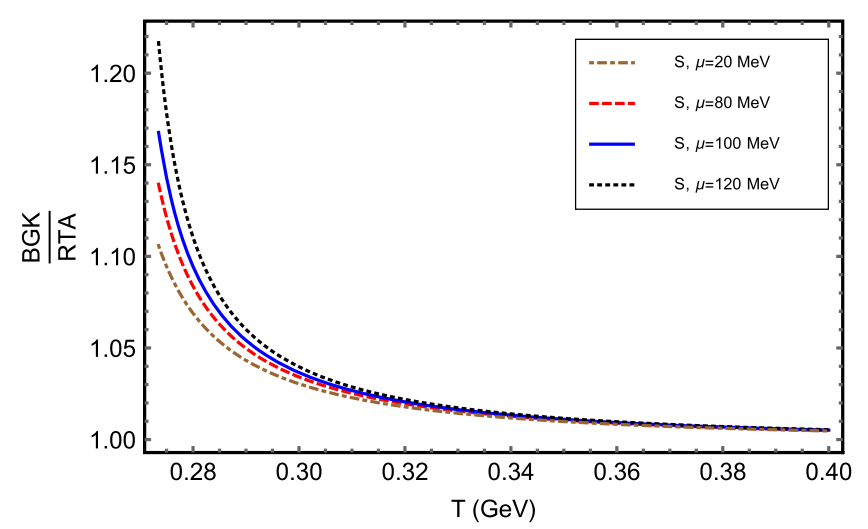

FIG. 1. Left: dependence of chemical potential and medium interactions on the temperature behavior of the Seebeck coefficient of the two-flavor QGP within the RTA. Right: The RTA results are compared with the estimations with the BGK collision kernel. 


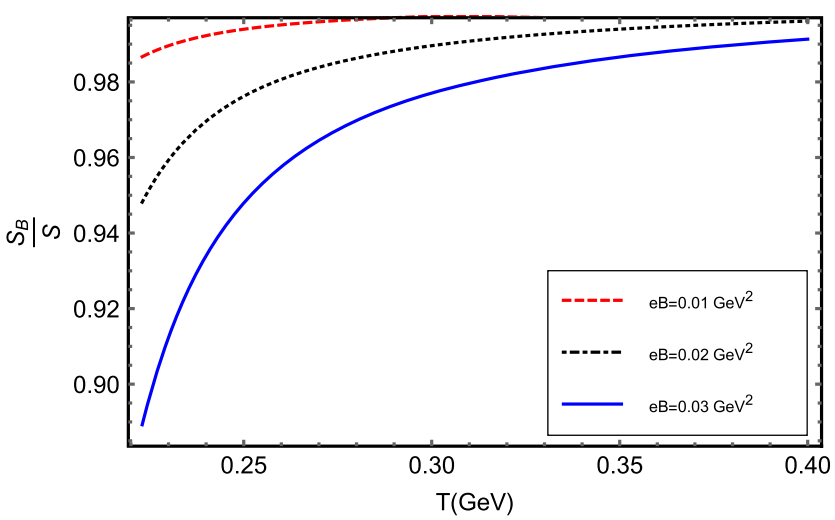

FIG. 2. Temperature dependence of Seebeck coefficient at $\mu=100 \mathrm{MeV}$ in a weakly magnetized medium.

described in Eq. (24). The first term of Eqs. (25) and (26) describes the RTA results, and other terms denote further corrections within the BGK description. For the quantitative estimation, the parameter $\nu$ is assumed as the thermal average of inverse of relaxation time for the elastic collisions. In the absence of a magnetic field, the choice of $\nu$ will not depend on the temperature behavior of the thermoelectric coefficient. It is observed that the collisional effect to the thermoelectric coefficient is more prominent in the temperature regime near $T_{c}$. The current density has contributions from the temperature gradient (with nonzero quark chemical potential) in the medium and also from the generated electric field. Both the contributions get corrections within the BGK analysis in comparison with the RTA result as described in Eq. (16). The collisional aspects of the medium are seen to be negligible at lower values of $\mu$. Notably, in the massless and ultrarelativistic limit $\left(z_{k} \rightarrow 1\right)$ limit, $S$ will be independent of the choice of collision integral, as the corrections to both contributions to the current density cancel exactly each other while defining the Seebeck coefficient.

The magnetic field induces anisotropy in the system and leads to a magnetic-field-dependent Seebeck coefficient and Nernst coefficient associated with the thermoelectric

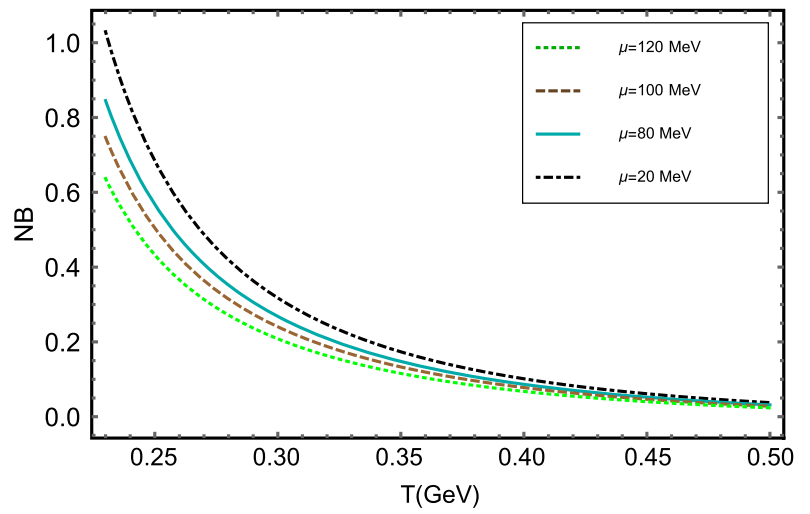

effect in the magnetized medium as described in Eqs. (47) and (48). The impact of the magnetic field on the Seebeck coefficient is shown in Fig. 2. In the weakly magnetized medium, the magnetic field dependence of the thermoelectric coefficient is entering through the Lorentz force via cyclotron frequency $\left|\Omega_{c k}\right|$. The effect of the magnetic field is observed to be more significant in the lower-temperature regimes. As the temperature increases, the particle motion is completely dominated by the temperature, and the effect of the magnetic field vanishes. However, in the temperature regimes near $T_{c}$ the Seebeck coefficient decreases with an increase in the strength of the field due to the factor $\frac{1}{1+\tau_{R_{k}}^{2} \Omega_{c k}^{2}}$. It is important to emphasize that Eq. (49) reduces back to Eq. (15) at the limit of $\mathbf{B}=0$.

The motion of the charged fermion gets deflected in the presence of a weak magnetic field due to the Lorentz force. The Hall-type conductivity associated with the thermal and electric charge transport in the hot QCD has been explored in Refs. [35,56]. The temperature variation of the Hall-type transport coefficient associated with the thermoelectric effect, Nernst coefficient, in the magnetized medium is depicted in Fig. 3. In contrast to the Seebeck coefficient, the Nernst coefficient is a positive quantity and is critically dependent on the quark chemical potential and strength of the magnetic field. It is seen that the $N|\mathbf{B}|$ decreases with an increase in chemical potential at a finite magnetic field. The dependence of the strength of the magnetic field on the Nernst coefficient is studied by plotting the ratio $\frac{N|\mathbf{B}|}{S}$ with the temperature in Fig. 3 (right). The ratio approaches zero asymptotically, which indicates that the impact of the magnetic field on the thermoelectric transport is negligible at sufficiently high temperature in the weakly magnetized QGP. However, the magnetic field effects on the Nernst coefficient are visible in the low-temperature regimes near $T_{c}$. Further, it is important to emphasize that the Nernst coefficient vanishes in a strongly magnetized medium due to the $1+1$-dimensional constraint motion of the charged particle.

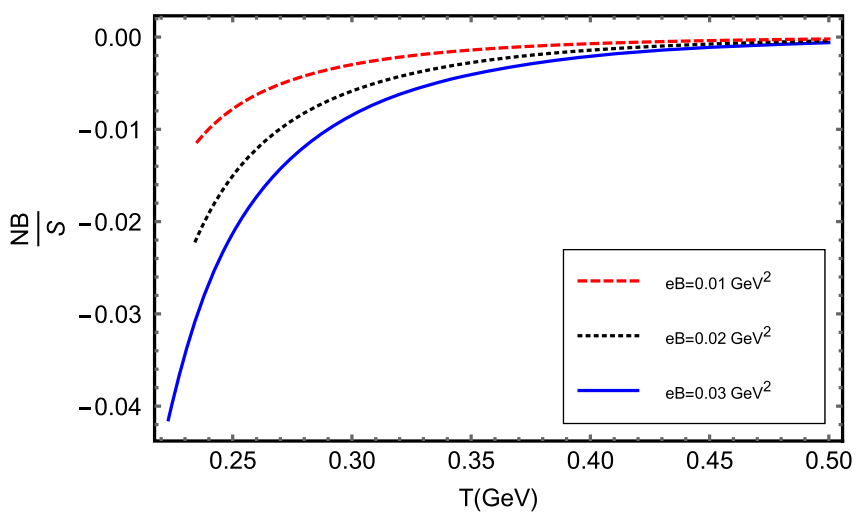

FIG. 3. Left: dependence of chemical potential on the temperature behavior of the Nernst coefficient at $|e B|=0.03$ GeV ${ }^{2}$. Right: the impact of the magnetic field on the Nernst coefficient at $\mu=100 \mathrm{MeV}$. 


\section{CONCLUSION AND OUTLOOK}

In this article, we have presented an analysis on the thermoelectric transport process and the associated transport coefficients in a collisional and magnetized hot QCD medium. The realistic EOS effects are embedded in the analysis within the framework of the EQPM through the temperature-dependent fugacity parameters. The nonequilibrium part of the momentum distribution of effective degrees of freedom is obtained by solving the effective transport equation within the EQPM employing an iterative Chapman-Enskog-like approach while choosing a proper collision kernel. The electric field generated due to temperature gradient in the hot QCD medium at finite quark chemical potential is quantified in terms of the Seebeck coefficient. The temperature behavior of the Seebeck coefficient in the collisional two-flavor QGP medium has been investigated. The effects of collision are incorporated in the analysis through the RTA and BGK collision kernels. The thermal medium interactions of the QCD medium are seen to have a significant impact on the thermoelectric behavior of the medium. The effects of the collisions and quark chemical potential are more visible in the temperature regimes near the transition temperature.

Further, we have studied the thermoelectric behavior of a weakly magnetized QCD matter. In the analysis, the temperature is considered to be the dominant energy scale in comparison with the strength of the magnetic field. The magnetic field induces anisotropy in the thermoelectric behavior of the medium. We have estimated the transport coefficients characterizing the thermoelectric behavior of the magnetized QCD medium, magnetic-field-dependent Seebeck and Nernst coefficients, within the effective transport equation. We have demonstrated the dependence of the magnetic field and quark chemical potential on thermoelectric coefficients in the weakly magnetized medium. The analysis is further extended to the strong magnetic field regime while considering the Landau level kinematics of the charged particle. Notably, the Nernst coefficient vanishes in a strongly magnetized medium due to the longitudinal motion of the particles.

The phenomenological aspects of the thermoelectric behavior may have a significant role in the baryon-rich medium and will be more relevant in the context of lowerenergy collisions probed in the RHIC beam energy scan and for upcoming experiments at Facility for Antiproton and Ion Research and in Nuclotron-based Ion Collider facility. The study of thermoelectric behavior of the collisional QCD medium in the presence of space-timevarying magnetic fields would be an interesting direction to work. The induced electric field from the decay of the magnetic field may put a constraint in the generated electric field from the temperature gradient at a finite chemical potential. A very recent study [71] proposed that temperature and chemical potential gradient in the heavy-ion collision experiment may induce spin Hall current. These aspects will be taken up for future investigations.

\section{ACKNOWLEDGMENTS}

The author would like to acknowledge Vinod Chandra for the immense encouragement, helpful discussions, and critical reading of the manuscript. The author acknowledges Indian Institute of Technology Gandhinagar for Institute postdoctoral fellowship. The author thanks the anonymous referee for the constructive inputs and suggestions.
[1] Adams et al. (STAR Collaboration), Nucl. Phys. A757, 102 (2005); K. Adcox et al. (PHENIX Collaboration), Nucl. Phys. A757, 184 (2005); B. B. Back et al. (PHOBOS Collaboration), Nucl. Phys. A757, 28 (2005); A. Arsence et al. (BRAHMS Collaboration), Nucl. Phys. A757, 1 (2005).

[2] K. Aamodt et al. (ALICE Collaboration), Phys. Rev. Lett. 105, 252301 (2010).

[3] C. Gale, S. Jeon, and B. Schenke, Int. J. Mod. Phys. A 28, 1340011 (2013).

[4] A. Jaiswal and V. Roy, Adv. High Energy Phys. 2016, 9623034 (2016), and the references therein.

[5] D. Teaney, Phys. Rev. C 68, 034913 (2003).

[6] P. Romatschke and U. Romatschke, Phys. Rev. Lett. 99, 172301 (2007).

[7] J. Adam et al. (ALICE Collaboration), Phys. Rev. Lett. 117, 182301 (2016).
[8] J. Adam et al. (ALICE Collaboration), Phys. Rev. Lett. 116, 132302 (2016).

[9] S. Acharya et al. (ALICE Collaboration), Phys. Rev. Lett. 125, 022301 (2020).

[10] J. Adam et al. (STAR Collaboration), Phys. Rev. Lett. 123, 162301 (2019).

[11] V. Skokov, A. Y. Illarionov, and V. Toneev, Int. J. Mod. Phys. A 24, 5925 (2009).

[12] W. Deng and X. Huang, Phys. Rev. C 85, 044907 (2012).

[13] K. Fukushima, D. E. Kharzeev, and H. J. Warringa, Phys. Rev. D 78, 074033 (2008); D. E. Kharzeev, L. D. McLerran, and H. J. Warringa, Nucl. Phys. A803, 227 (2008); D. E. Kharzeev, Ann. Phys. (Amsterdam) 325, 205 (2010); D. E. Kharzeev and D. T. Son, Phys. Rev. Lett. 106, 062301 (2011).

[14] A. V. Sadofyev and M. V. Isachenkov, Phys. Lett. B 697, 404 (2011); A. V. Sadofyev, V. I. Shevchenko, and V. I. Zakharov, Phys. Rev. D 83, 105025 (2011). 
[15] K. Fukushima, K. Hattori, H. U. Yee, and Y. Yin, Phys. Rev. D 93, 074028 (2016).

[16] B. Singh, S. Mazumder, and H. Mishra, J. High Energy Phys. 05 (2020) 068.

[17] M. Hasan, B. Chatterjee, and B. K. Patra, Eur. Phys. J. C 77, 767 (2017).

[18] B. Singh, L. Thakur, and H. Mishra, Phys. Rev. D 97, 096011 (2018).

[19] A. Bandyopadhyay, C. A. Islam, and M. G. Mustafa, Phys. Rev. D 94, 114034 (2016); A. Das, N. Haque, M. G. Mustafa, and P. K. Roy, Phys. Rev. D 99, 094022 (2019).

[20] S. Li, K. A. Mamo, and H. U. Yee, Phys. Rev. D 94, 085016 (2016).

[21] A. Bandyopadhyay, B. Karmakar, N. Haque, and M. G. Mustafa, Phys. Rev. D 100, 034031 (2019); B. Karmakar, R. Ghosh, A. Bandyopadhyay, N. Haque, and M. G. Mustafa, Phys. Rev. D 99, 094002 (2019).

[22] S. Koothottil and V. M. Bannur, Phys. Rev. C 99, 035210 (2019).

[23] J. Dey, S. Satapathy, A. Mishra, S. Paul, and S. Ghosh, arXiv:1908.04335; J. Dey, S. Satapathy, P. Murmu, and S. Ghosh, arXiv:1907.11164.

[24] K. Tuchin, Phys. Rev. C 88, 024911 (2013); 93, 014905 (2016).

[25] L. McLerran and V. Skokov, Nucl. Phys. A929, 184 (2014).

[26] S. Mitra and V. Chandra, Phys. Rev. D 96, 094003 (2017).

[27] P. Kalikotay, N. Chaudhuri, S. Ghosh, U. Gangopadhyaya, and S. Sarkar, Eur. Phys. J. A 56, 79 (2020).

[28] R. Marty, E. Bratkovskaya, W. Cassing, J. Aichelin, and H. Berrehrah, Phys. Rev. C 88, 045204 (2013).

[29] G. P. Kadam, H. Mishra, and L. Thakur, Phys. Rev. D 98, 114001 (2018).

[30] P. Deb, G. P. Kadam, and H. Mishra, Phys. Rev. D 94, 094002 (2016).

[31] M. Greif, F. Reining, I. Bouras, G. Denicol, Z. Xu, and C. Greiner, Phys. Rev. E 87, 033019 (2013).

[32] D. Fernandez-Fraile and A. Gomez Nicola, Eur. Phys. J. C 62, 37 (2009).

[33] B. Feng, Phys. Rev. D 96, 036009 (2017).

[34] A. Das, H. Mishra, and R. K. Mohapatra, Phys. Rev. D 101, 034027 (2020); 99, 094031 (2019).

[35] A. Das, H. Mishra, and R. K. Mohapatra, Phys. Rev. D 100, 114004 (2019).

[36] L. Thakur and P. K. Srivastava, Phys. Rev. D 100, 076016 (2019).

[37] S. Ghosh, A. Bandyopadhyay, R. L. S. Farias, J. Dey, and G. Krein, Phys. Rev. D 102, 114015 (2020).

[38] B. Chatterjee, R. Rath, G. Sarwar, and R. Sahoo, Eur. Phys. J. A 57, 45 (2021).

[39] S. Rath and B. K. Patra, Phys. Rev. D 100, 016009 (2019); arXiv:2005.00997.

[40] K. K. Gowthama, M. Kurian, and V. Chandra, arXiv: 2012.07156

[41] M. Matusiak, K. Rogacki, and T. Wolf, Phys. Rev. B 97, 220501(R) (2018).
[42] M. M. Wysokinski and J. Spalek, J. Appl. Phys. 113, 163905 (2013).

[43] J. R. Bhatt, A. Das, and H. Mishra, Phys. Rev. D 99, 014015 (2019).

[44] A. Abhishek, A. Das, D. Kumar, and H. Mishra, arXiv:2007.14757.

[45] A. Das, H. Mishra, and R. K. Mohapatra, Phys. Rev. D 102 , 014030 (2020).

[46] H. X. Zhang, J. W. Kang, and B. W. Zhang, arXiv:2004 .08767

[47] D. Dey and B. K. Patra, Phys. Rev. D 102, 096011 (2020).

[48] V. Chandra and V. Ravishankar, Phys. Rev. D 84, 074013 (2011); V. Chandra, R. Kumar, and V. Ravishankar, Phys. Rev. C 76, 054909 (2007).

[49] M. Kurian and V. Chandra, Phys. Rev. D 96, 114026 (2017).

[50] S. Mitra and V. Chandra, Phys. Rev. D 97, 034032 (2018).

[51] A. Czajka, S. Hauksson, C. Shen, S. Jeon, and C. Gale, Phys. Rev. C 97, 044914 (2018).

[52] S. Plumari, A. Puglisi, F. Scardina, and V. Greco, Phys. Rev. C 86, 054902 (2012).

[53] A. Jaiswal, R. Ryblewski, and M. Strickland, Phys. Rev. C 90, 044908 (2014).

[54] A. K. Panda, A. Dash, R. Biswas, and V. Roy, arXiv:2011 .01606 .

[55] S. Bhadury, W. Florkowski, A. Jaiswal, and R. Ryblewski, Phys. Rev. C 102, 064910 (2020).

[56] M. Kurian, Phys. Rev. D 102, 014041 (2020).

[57] J. L. Anderson and H. R. Witting, Physica (Amsterdam) 74, 466 (1974).

[58] K. Hattori, X. G. Huang, D. H. Rischke, and D. Satow, Phys. Rev. D 96, 094009 (2017); K. Hattori and D. Satow, Phys. Rev. D 94, 114032 (2016).

[59] A. Hosoya and K. Kajantie, Nucl. Phys. B250, 666 (1985).

[60] A. Jaiswal, Phys. Rev. C 87, 051901 (2013).

[61] B. Schenke, M. Strickland, C. Greiner, and M. H. Thoma, Phys. Rev. D 73, 125004 (2006).

[62] A. Kumar, M. Y. Jamal, V. Chandra, and J. R. Bhatt, Phys. Rev. D 97, 034007 (2018).

[63] S. A. Khan and B. K. Patra, arXiv:2011.02682.

[64] B. F. Jiang, D. F. Hou, and J. R. Li, Phys. Rev. D 94, 074026 (2016).

[65] M. E. Carrington, T. Fugleberg, D. Pickering, and M. H. Thoma, Can. J. Phys. 82, 671 (2004).

[66] A. Ayala, C. A. Dominguez, S. Hernandez-Ortiz, L. A. Hernandez, M. Loewe, D. Manreza Paret, and R. Zamora, Phys. Rev. D 98, 031501 (2018).

[67] A. Dash, S. Samanta, J. Dey, U. Gangopadhyaya, S. Ghosh, and V. Roy, Phys. Rev. D 102, 016016 (2020).

[68] M. Kurian, S. Mitra, S. Ghosh, and V. Chandra, Eur. Phys. J. C 79, 134 (2019).

[69] K. Fukushima and Y. Hidaka, Phys. Rev. Lett. 120, 162301 (2018).

[70] M. Kurian and V. Chandra, Phys. Rev. D 99, 116018 (2019).

[71] S. Y. F. Liu and Y. Yin, arXiv:2006.12421. 